\title{
Augmentation of anti-bacterial efficacy of Augmentin- an antibiotic by Foeniculum vulgare extract
}

\author{
Sumera Javad ${ }^{1 *}$, Ammara Tahir ${ }^{1}$, Nadia Ghaffar ${ }^{1}$, Saiqa Ilyas ${ }^{2}$ and \\ Iqra Akhtar ${ }^{1}$ \\ 1. Department of Botany, Lahore College For Women University, Lahore-Pakistan \\ 2. Department of Biotechnology, Lahore College For Women University, Lahore-Pakistan \\ *Corresponding author's email: zif 4@yahoo.com
}

Citation

Sumera Javad, Ammara Tahir, Nadia Ghaffar, Saiqa Ilyas and Iqra Akhtar. Augmentation of anti-bacterial efficacy of Augmentin- an antibiotic by Foeniculum vulgare extract. Pure and Applied Biology. Vol. 8, Issue 2, pp1818-1823. http://dx.doi.org/10.19045/bspab.2019.80125

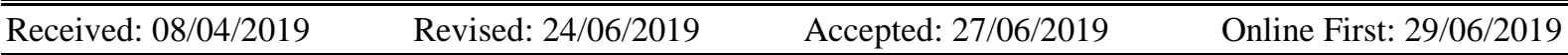

\section{Abstract}

Plant extracts in combination with antibiotics propose an innovative approach to treat a number of infectious diseases. Present research was carried out to evaluate the anti-bacterial activity of hexane, methanol and ethyl acetate fractions of water extract from Foeniculum vulgare in combination with antibiotic Augmentin. Plant extract and anti-bacterial (Augmentin) were used as control. For anti-bacterial assay, agar well diffusion method was used against selected bacterial strains i.e. Bacillus cereus, Pseudomonas aeruginosa and Brucella melitensis. Results showed that methanolic and ethyl acetate fractions, in combination with antibiotic, increased its efficiency against $P$. aeruginosa. Furthermore, n-hexane fraction, in combination with antibiotic, increased the efficiency of antibiotic against Bacillus sp. The results of each fraction confirmed that active substances of plant were involved in augmentation of antibiotic. Methanolic and ethyl acetate fractions were found to be rich in phenolics while n-hexane fraction was rich in terpenes. The results provided support for $F$. vulgare to be used in traditional medicines for the treatment of various infectious diseases and for commercial level augmentation of antibiotics to produce new antibiotics.

Keywords: Antibacterial activity; Augmentation; Augmentin; F. vulgare

\section{Introduction}

Pathogenic bacteria are causing health concerns to modern world at a very fast pace. Synthetic antibiotics are considered very efficient and effective to control the infectious diseases. But application of synthetic antibiotics has developed the resistant strains of bacteria. Synthetic antibiotics have other associated problems as well, like accumulation in food chain, high and acute toxicity, long degradation period and extension of their power to destroy both useful and harmful bacteria. Furthermore it takes a lot of time and effort to develop and introduce a new antibiotic to human race $[1,2]$. Therefore great attention is dedicated to the production of new antibacterial agents which are used in the control of pathogenic microorganisms. Different studies provide evidence that some aromatic plants might indeed be potential and valuable sources of natural antibiotics [3]. Plants produce antimicrobial secondary metabolites in response to pathogen attack or abiotic stress. Plant secondary metabolites are the part of plant's defense system against insects, herbivores and microorganisms [4]. 
Globally interest has increased towards the development of new antibiotics due to the emergence of resistant strains of microbes. In this regards plant secondary metabolites are gaining attention for their biological activities. Therefore plants are being selected to fight against resistant strains of bacteria. In most parts of the world medicinal plants are already in use due to their antiviral, antibacterial and antifungal activities [5]. There are a number of plants like garlic, curry, basil, cinnamon, ginger, black pepper which have pronounced antimicrobial activities $[6,7]$. F. vulgare Mill. (English - Fennel) belonging to the family Apiaceae, possesses great medicinal properties. The roots are used as purgative and diuretic. The fruits are sweet, acrid, bitter, anthelmintic, carminative, digestive and stomachic. The seed extracts are used in cardiac diseases [8]. In present study polar and non-polar fraction of microwave assisted water extract of $F$. vulgare were evaluated for their antibacterial activity. These fractions were also used in combination with antibiotic "Augmentin" to check their synergistic effect with the antibiotic. Their antibacterial efficiency was tested against Bacillus cereus, Pseudomonas aeruginosa and Brucella melitensis. The main objectives of the study were to extract the plant metabolites from $F$. vulgare, to evaluate the antibacterial properties of different fractions of $F$. vulgar Mill. and their synergistic effect with Augmentin against the selected pathogenic bacteria.

\section{Materials and methods}

F. vulgare Mill. Seeds were provided by NARC (National Agricultural Research Council) Islamabad, Pakistan. Plant material was powdered by using electric grinder. Powdered fennel was placed in air tight jars, properly labeled and used later on for extraction purpose.

\section{Extraction by Microwave assisted extraction}

Plant material was extracted using microwave assisted extractor (Saneo, MDS-6G) at $800 \mathrm{~W}$ power level for 5 minute using water as a solvent and temperature was maintained at $80^{\circ} \mathrm{C}$. Extract was filtered in pre-weighed beaker. Plant extract was fractionated with selected solvents (n-hexane, ethyl acetate and methanol) in separating funnel in order of polarity. For this fractionation, $50 \mathrm{~mL}$ of plant extract was taken in separating funnel and $30 \mathrm{~mL}$ of $\mathrm{n}$-hexane was added, shaken and left for 2 hours. Two layers were formed in separating funnel. Lower layer (water fraction) and upper n-hexane layer were separated in pre-weighed separate beakers. It was repeated 3-4 times to get maximum n-hexane fraction. Same procedure was repeated with other two solvents. Each sample was made in three replicates. Afterwards all fractions were dried with the help of rotary evaporator. In this way 3 samples (n-hexane, Methanol and Ethyl fraction) were obtained for antimicrobial activity.

\section{Antimicrobial activity}

$0.5 \mathrm{~g}$ of each dried material was taken in separate vial and $1 \mathrm{~mL}$ of respective solvent was added to each vial. In separate vial, $0.1 \mathrm{~g}$ Augmentin and $1 \mathrm{~mL}$ solvent was added to be used as a standard antibiotic. In this way 4 samples (methanol fraction, ethyl acetate fraction, $\mathrm{n}$-hexane fraction and Augmentin) were obtained.

Properly identified bacterial strains of Bacillus cereus, Pseudomonas aeruginosa, and Brucella melitensis were taken from Biotechnology Department, Lahore College for Women University Lahore, Pakistan. Glassware was dried and sterilized in air dry oven at $170^{\circ} \mathrm{C}$ for 2 hours. Media was prepared by adding $6.25 \mathrm{~g}$ of LB nutrient medium, $3.5 \mathrm{~g}$ of bacteriological agar and $250 \mathrm{ml}$ of double distilled water in $500 \mathrm{ml}$ conical flask. The media was autoclaved at $15 \mathrm{Ib} /$ inches $^{2}$ for $20 \mathrm{~min}$ at $121^{\circ} \mathrm{C}$ and poured into sterilized petri plates. Solidified media was inoculated with cultures of bacterial strains. Using a sterilized cork borer, wells were made in solidified nutrient agar medium. The extracts were poured (with the help of dropper) in the wells and all the plates were 
incubated for 24 hours at $37^{\circ} \mathrm{C}$. The antibacterial activity was recorded by measuring the diameter of inhibition zone formed as clear region around the well.

\section{Phytochemical analysis of fractions Estimation of total phenolics}

Spectrophotometric analysis was used to estimate the total phenolics present in the seed extract of $F$. vulgare. Sample was prepared by mixing $1 \mathrm{mg}$ of dried plant extract with $1 \mathrm{~mL}$ of methanol and $1 \mathrm{~mL}$ of FC reagent (Folin Ciocalteu reagent). After three minutes of sample making, $1 \mathrm{~mL}$ of concentrated sodium carbonate $\left(\mathrm{Na}_{2} \mathrm{CO}_{3}\right)$ solution was added and mixed well. The reaction mixture was kept in dark for $1 \mathrm{~h}$ and 30minutes. After this incubation time, the absorbance of the reaction mixture was read at $725 \mathrm{~nm}$. Total phenolic content was calculated by plotting the values of absorption of samples against standard curve of Gallic acid.

\section{Estimation of total flavonoid content}

Again spectrophotometric assay was used to estimate the total Flavonoid content present in the plant extracts. A little amount of plant extract $(250 \mu \mathrm{L})$ in concentration of $1 \mathrm{mg} / \mathrm{mL}$ was mixed with $1.25 \mathrm{~mL}$ of distilled water and $75 \mu \mathrm{L}$ of $5 \%$ Sodium nitrite $\left(\mathrm{NaNO}_{2}\right)$ solution. This mixture was incubated for 5 minutes. After 5 minutes, $150 \mu \mathrm{L}$ of $10 \%$ of Aluminum Chloride $\left(\mathrm{AlCl}_{3}\right)$ solution was mixed in the reaction mixture. The reaction mixture was once again incubated for 6 minutes, after which $500 \mu \mathrm{L}$ of $1 \mathrm{M}$ Sodium hydroxide $(\mathrm{NaOH})$ and $275 \mu \mathrm{L}$ of distilled water were added to the reaction mixture. The reaction mixture was mixed thoroughly and absorbance was checked at wavelength of $510 \mathrm{~nm}$. The total flavonoid content was calculated by plotting the values of absorbance against the standard curve of Catechin.

\section{Estimation of terpenes}

For estimation of terpenes $1 \mathrm{~g}$ of each plant sample was taken and mixed with chloroform and then added the conc. $\mathrm{H}_{2} \mathrm{SO}_{4}$ drop wise. Formation of ring in the middle indicated the presence of terpenes while intensity of color of that ring showed the amount of terpenes.

\section{Statistical analysis}

Numerical values for zone of inhibition were calculated and noted. The data were analyzed by one way analysis of variance (ANOVA) and SPSS computer software was used. Tukey's test was used as post hoc test at 5\% significance level.

\section{Results and discussion}

All the extract and antibiotic combinations showed a higher inhibition zone (IZ) against bacterial strain as compared to pure plant extract. Methanolic fraction of $F$. vulgare water extract was used against three bacterial species $P$. aeruginosa, $B$. melitensis, B. cereus. After 24 hours significant inhibition zones were observed which showed the antibacterial activity against all species (Figure 1a). Maximum enhancement of antibacterial activity of antibiotic by plant extract was observed against $P$. aeruginosa. Methanolic fraction of $F$. vulgare increased the inhibition zone of Augmentin against $P$. aeruginosa from $25.23 \mathrm{~mm}$ to $35.5 \mathrm{~mm}$ (Figure $1 \mathrm{~b}$ ). But it did not work with Augmentin against other two bacterial strains.

F. vulgare ethyl acetate extract was also used to determine anti-bacterial activity against selected bacterial strains (Figure 2a). Antibacterial activity of ethyl acetate fraction of fennel water extract, ethyl acetate fraction+augmentin and augmentin was also checked and inhibition zones were measured. Maximum zone of inhibition observed was $40.60 \mathrm{~mm}$ against $P$. aeruginosa as compared to Bacillus sp. and Brucella sp. which has inhibition zone of $30.56 \mathrm{~mm}$ and $30.26 \mathrm{~mm}$ respectively. Results showed that ethyl acetate fractions significantly enhanced the antibacterial activity of antibiotic against $P$. aeruginosa (Figure 2b). 


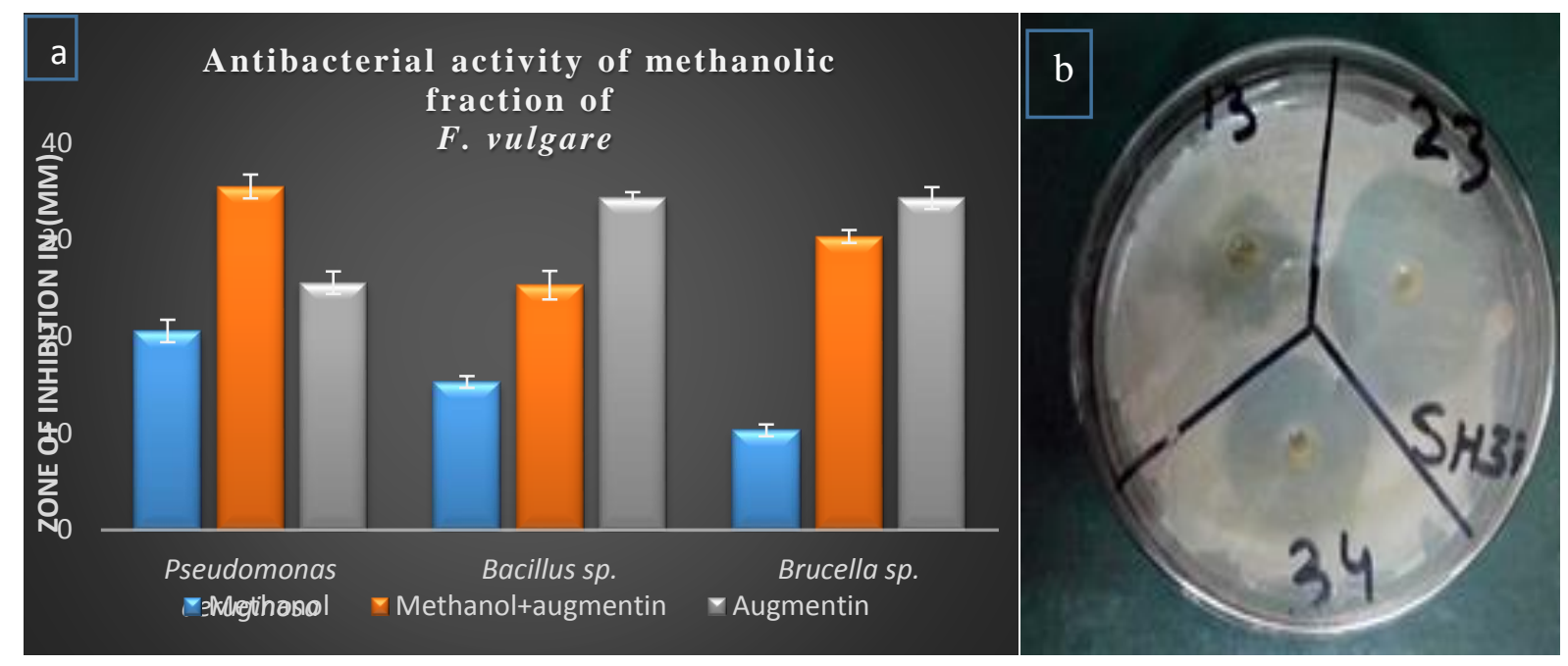

Figure 1. (a) Antibacterial activity of Methanolic fraction of $F$. vulgare against $P$. aeruginosa, Bacillus sp. and Brucella sp. (b) Antibacterial activity of methanol fraction (13), methanol fraction+augmentin (23) and augmentin (34) against $P$. aeruginosa.

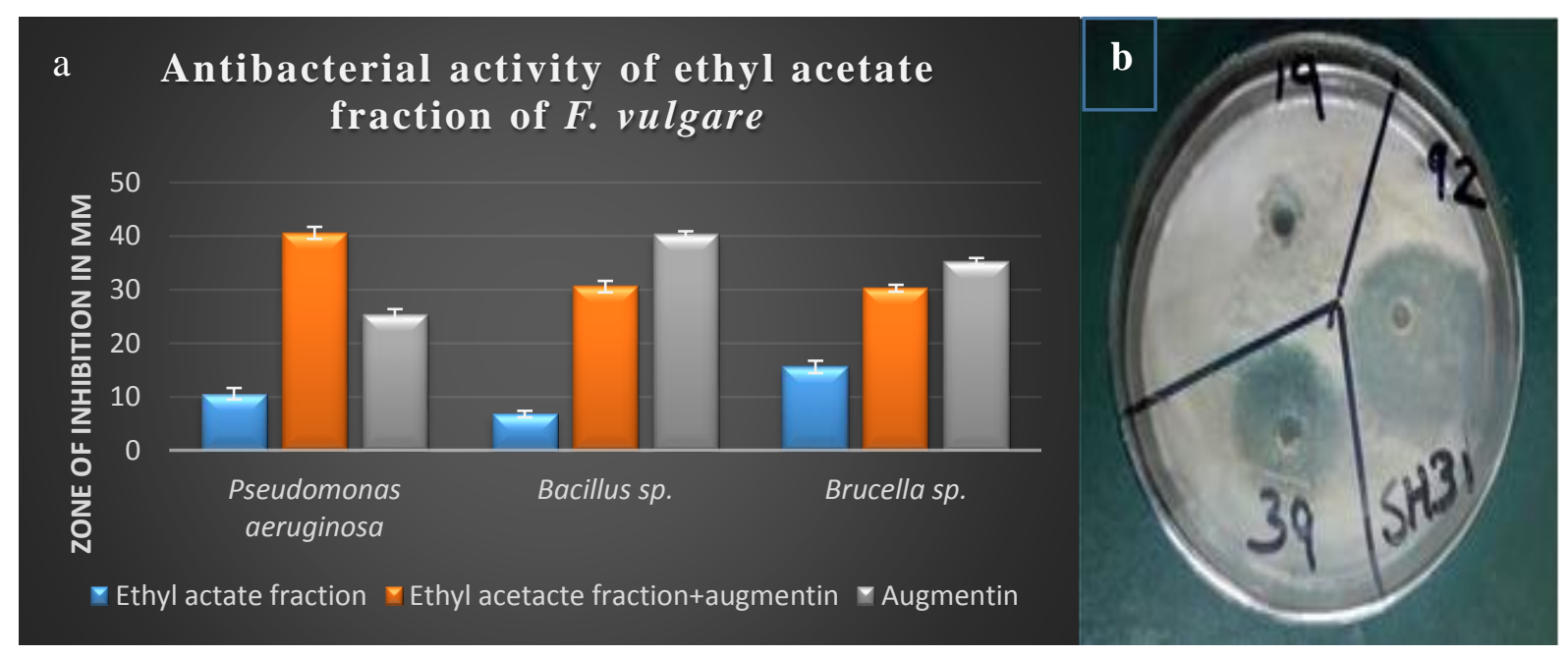

Figure 2. (a) Antibacterial activity of ethyl acetate fraction of $F$. vulgare against $P$. aeruginosa, Bacillus sp. and Brucella sp. (b) Antibacterial activity of ethyl acetate fraction (19), ethyl acetate fraction+augmentin (92) and augmentin (39) against $P$. aeruginosa

Antibacterial activity of $n$-hexane fraction of fennel water extract was also evaluated against $P$. aeruginosa, $B$. melitensis, $B$. cereus. After 24 hours inhibition zones were measured which showed that nhexane fraction of fennel water extract increased the antibacterial effect of augmentin against $B$. cereus (Figure $3 a$ ). But it did not have synergic effect against other two bacterial strains used (Figure $3 b$ ).
Phytochemical analysis of fractions of fennel water extract showed significant differences in chemical composition. Methanolic and ethyl acetate fractions showed higher amounts of phenolics and flavonoids; and no or little terpenes over all while n-hexane fraction showed higher amount of terpenes but very less phenolics and flavonoids (Table 1). 

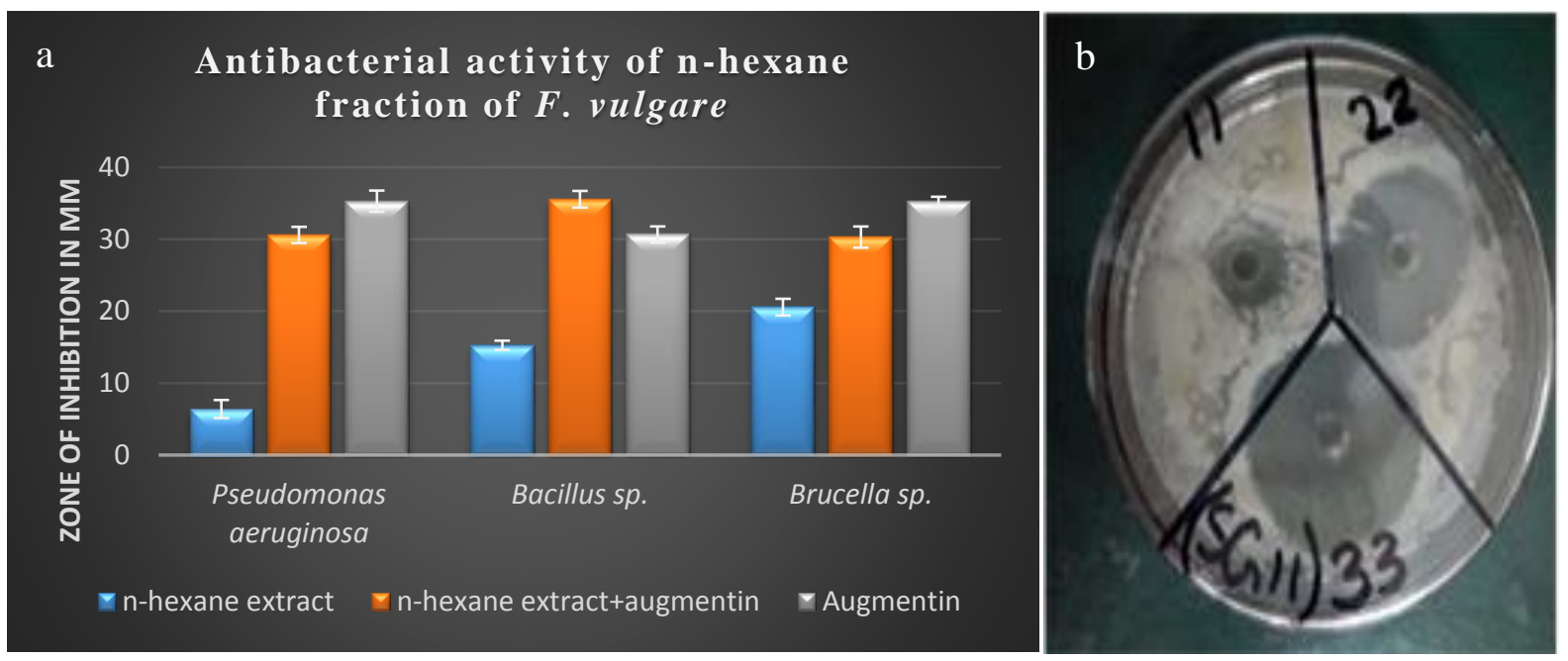

Figure 3. (a) Antibacterial activity of $n$-hexane fraction of $F$. vulgare against $P$. aeruginosa, Bacillus sp. and Brucella sp. (b) Antibacterial activity of n-hexane fraction(11), n-hexane fraction+augmentin (22) and augmentin (33) against Brucella $\mathbf{s p}$

Table 1. Phytochemical analysis of different fractions of water extract of Foeniculum vulgare Mill

\begin{tabular}{|c|c|c|c|}
\hline Plant fraction & Phenolics & Flavonoids & Terpenes \\
\hline Methanolic & +++ & +++ & -- \\
\hline Ethyl acetate & +++ & + & + \\
\hline n-hexane & + & + & +++ \\
\hline
\end{tabular}

+++ Significantly higher quantities; +Present in very less amount, ---Absent

\section{Discussion}

In present study three different fractions of $F$. vulgare water extract were used (methanol, $\mathrm{n}$ hexane and ethyl acetate) individually and in combination with antibiotic (Augmenton) against $B$. cereus, $P$. aeruginosa and $B$. melitensis. Results showed that methanolic and ethyl acetate extract in combination with antibiotic increased the efficiency of antibiotic against Pseudomonas aeruginosa (with $35.50 \mathrm{~mm} \mathrm{IZ} \mathrm{and} 40.60 \mathrm{~mm}$ IZ respectively) while $n$-hexane fraction in combination with antibiotic increased the efficiency of antibiotic against Bacillus sp. (35.56mm IZ).

It has been postulated that effectiveness of plant extract as antibacterial is due to presence of different chemical compounds. These chemical compounds include flavonoids, alkaloids, terpenoids, essential oils which are classified as active antimicrobial agents $[9,10]$.

Plant secondary metabolites when used in combination with antibiotics, offer opportunities of fast cure, lesser toxicity, and broader spectrum of action and finally increase in the efficiency of antibiotics [11]. A precise blend of known antibiotics and plant extracts may be a novel method to treat the diseases caused by the resistant strains. This is also called synergism in which combined effect of two substances is better than their individual effect. Plant extract may play their role in increasing the permeability and perturbation of the cell walls and cell membranes. Due to this increased permeability, influx of antibiotics into bacterial cell may be increased. This may also be attributed to some chemical reactions of plant secondary metabolites like phenolics and flavonoids with antibiotics or microbes. In such types of proposed reactions, for example, plant metabolites may form a bond with some specific biochemical of bacteria which otherwise help them in multiple drug resistance. Such plant secondary metabolites are reported to be very diverse in their synergic effect with antibiotics. They are not specific in action towards any class of bacteria or antibiotics [12]. There are a number of advantages associated with such synergism of antibiotics and plant extracts. It increases the efficiency of existing antibiotics with least undesirable side effects. It adds natural substances to the therapy instead of new synthetic molecules which are usually 
not acceptable by the human body. It may also increase the bioavailability of some compounds which otherwise were not such efficient. It reduces the dose requirement of the antibiotic. It has enhanced therapeutic effect as compared to the synthetic antibiotics $[13,14]$.

\section{Conclusion}

It is concluded from the present study that the presence of antimicrobial agents like phenolics, flavonoids and terpens in $F$. vulgare extract and its synergism with antibiotic Augmentin gives support to its traditional use for treating conditions associated with microorganisms in humans. It is also concluded that $F$. vulgare extract also helped the Augmentin to fight against multi resistant microbes and community acquired infections which is a step towards more effective, less toxic and cost effective new antibiotics.

\section{Authors' contributions}

Designed the experiment: S Javad, Performed the experiments: A Tahir \& N Ghaffar, Analyzed the data: S Ilyas, Wrote the paper: $\mathrm{N}$ Ghaffar \& I Akhtar.

\section{Acknowledgement}

Research work was done under the research project of Higher Education Pakistan, NRPU program, funding number 203545/NRPU/R\&D/HEC/14/883.

\section{References}

1. Otshudi AL, Foriers A, Vercruysse A, Van Zeebroeck A \& Lauwers S (2000). In vitro antimicrobial activity of six medicinal plants traditionally used for the treatment of dysentery and diarrhoea in Democratic Republic of Congo (DRC). Phytomedicine 7: 167-172.

2. Chand H (2017). Antimicrobial resistance and the alternative resources with special emphasis on plants based antimicrobials. Review Plants 6: 1-11.

3. Balandrin MF, Klocke J, Wurtele ES \& Bollinger WH (1985). Natural plant chemicals: sources of industrial and medicinal materials. Amer Asso Adv of Sci 228: 1154-1159.

4. Bai VR \& Kanimozhi D (1990). Evaluation of Anti Microbial Activity of
Coriandrum sativum. Int J Sci Res Rev 1: 01-10.

5. Caceres A, Cano O, Samayoa B \& Aguilar L (1990). Plants used in Guatemala for the treatment of gastrointestinal disorders. $J$ of Ethnopharm 30: 55-73.

6. Cowan MM (1990). Plant products as antimicrobial agents. Clin Microb Rev 12: 564-582.

7. Gupta PD \& Birdi TJ (2017). Development of Botanicals to combat antibiotic resistance. J of AIM 5: 266-275.

8. Sastri BN (1962). The Wealth of India. A Dictionary of Indian Raw Materials and Industrial Products. Raw Materials 6: 483.

9. Sibanda T \& Okoh AI (2007). The challenges of overcoming antibiotic resistance: Plant extracts as potential sources of antimicrobial and resistance modifying agents. Afr $J$ of Biotech 6: 2886-2896.

10. Shahid W, Durrani R, Iram S, Durrani M \& Khan FA (2013). Antibacterial activity in vitro of medicinal plants. Sky J Microb Res 1: 5-21.

11. Essawi T \& Srour M (2000). Screening of some Palestinian medicinal plants for antibacterial activity. J of Ethnopharm 70: 343-349.

12. Olajuyigbe OO \& Afolayan AJ (2012). Synergistic interactions of methanolic extract of Acacia mearnsii De Wild. with antibiotics against bacteria of clinical relevance. Inter J of Mol Sci 13: 89158932.

13. Rojas A, Hernandez L, Pereda-Miranda R \& Mata R (1992). Screening for antimicrobial activity of crude drug extracts and pure natural products from Mexican medicinal plants. $J$ of Ethnopharm 35: 275-283.

14. Akujobi C, Anyanwu B, Onyeze C \& Ibekwe V (2004). Antibacterial activities and preliminary phytochemical screening of four medicinal plants. J Appl Sci 7: 4328-4338. 